\title{
Risk of Ovarian Cancer After Treatment for Infertility
}

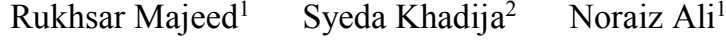 \\ 1.MsDU, University of Lahore, Pakistan \\ 2.Mphill (ultrasound),PHD*,University of Lahore, Pakistan
}

\begin{abstract}
Background: Ovarian cancer is the most frequent cause of death from gynecologic malignancies in the world. Ovarian cancer is predominantly a disease of postmenopausal period, with a median age at diagnosis of 59 years. In recent years, there have been many debates about the relationship between fertility medication and cancer. Due to the lack of comprehensive study of this matter, and as understanding the relationship between the use of fertility drugs and cancer is of importance, the present study was conducted to investigate the relationship between infertility drugs and cancer in women. Objective: To determine the risk of ovarian cancer after treatment of infertility. Study Selection: Multiple articles were reviewed. Prospective studies and case reports were excluded from the data. Retrospective studies were included in the study. Methods and Materials: A review of the scientific literature concerning the association between the use of fertility treatments and the risk of ovarian cancer was done. In this study, digital databases including PubMed, EMBASE and Google scholar were searched. The survey was carried out using keywords such as "infertility", " ovarian cancer risk", "gynecological cancer", "gynecological cancer risk", "cancer risk", "in vitro fertilization", "progesterone", "fertility drugs", "infertility treatment", variously associated together. Results: Using the search criteria, 25 researches were examined based on the title and abstract. All the 25 studies were considered in their full versions. Of these works, including literature reviews or meta-analysis reports, it was concluded that infertility treatment has a positive relationship with ovarian cancer. Conclusion: The results of this study suggest that fertility drug usage significantly contribute to overall risk of ovarian cancer when adjusting for known confounding factors. In the next years, the incidence of female infertility is expected to increase. A lot of new drugs are under investigation while other recent drugs are already in current use. More study can be done so that the mortality and mobility could be reduced in the society.
\end{abstract}

Keywords: infertility, fertility drugs, ovarian cancer, infertility treatment

DOI: $10.7176 / \mathrm{JHMN} / 82-09$

Publication date: November $30^{\text {th }} 2020$

\section{Introduction:}

Infertility is a common condition, which affects $\sim 10-15 \%$ of all couples in the world. ${ }^{1}$ During the past three decades, the use of fertility treatments, including intrauterine insemination, in vitro fertilization (IVF) etc. has increased constantly in western countries. ${ }^{2}$ The increased use of fertility drugs has given rise to studies of their potential adverse health effects and, especially, their potential to cause cancer. ${ }^{3}$ Several epidemiological studies addressed the potential connection between the use of fertility drugs and risk for ovarian cancer. Ovarian cancer is multifactorial and complex in etiology. Lifestyle factors shown to increase the risk of ovarian cancer include low parity ${ }^{4}$, late onset of menopause ${ }^{5}$, and perineal talc use. ${ }^{6}$ Oral contraceptive use, breastfeeding, and tubal ligation have been shown to have a protective effect on ovarian cancer risk. ${ }^{7}$ Several theories have been proposed to explain the mechanisms by which these factors affect risk of ovarian cancer. ${ }^{8}$ The incessant ovulation hypothesis theorizes that the repeated damage and subsequent repair cycles that occur during ovulation on the epithelial surface of the ovary contributes to DNA damage and increases the risk of developing ovarian cancer. ${ }^{9}$ The gonadotropin hypothesis postulates that exposure to high levels of circulating pituitary gonadotropins, which stimulates the ovarian surface epithelium, plays a role in the development of ovarian cancer. ${ }^{10}$ Both of these theories suggest that the use of fertility drugs, which often contain gonadotropins and stimulate ovulation, may increase the risk of ovarian cancer. Fertility drug use has increased markedly in the world. The use of infertility services was more common among older women and women who were childless. Despite the growing number of women seeking fertility treatment, the effects of fertility drug use on ovarian cancer risk remain uncertain. ${ }^{11}$

The increasing use of fertility drugs necessitates the separation of the effects of underlying infertility and other confounding factors from those of fertility drug use. This study is one of the largest case-control studies of ovarian cancer conducted to date. The aim is to determine the risk of ovarian cancer after treatment of infertility.

\section{Study Selection}

Multiple articles were reviewed. Prospective studies and case reports were excluded from the data. Retrospective studies were included in the study. 


\section{Results}

Using the search criteria, 25 researches were examined based on the title and abstract. All the 25 studies were considered in their full versions. Of these works, including literature reviews or meta-analysis reports, it was concluded that infertility treatment has a positive relationship with ovarian cancer.

\section{Discussion}

This study shows that there is a positive relationship between fertility drugs and ovarian cancer. Early studies that reported an increased risk of ovarian cancer among fertility drug users included small numbers of ovarian cancer patients exposed to fertility drugs and were unable to adjust for risk factors known to impact ovarian cancer risk. ${ }^{12}$ A previous study showed that the infertile women in cohort are at a higher risk of ovarian cancer than women in the general Danish population, even after adjustment for parity. ${ }^{13}$

The collaborative analysis of 12 case-control studies in the United States 5 suggested that infertile women who received fertility drugs but remained nulliparous may be at particularly high risk. This result is in line with those of two subsequent studies that found statistically non-significantly increased risks among women who remained nulliparous. However, our study, in line with others, found no association between overall risk of ovarian cancer and use of fertility drugs after stratifying for parity. ${ }^{14}$

In a retrospective study Brinton et al. evaluated 12193 infertile women followed for a median of 18.8 years and reported 45 ovarian cancers. This study used a detailed collection of information about drug exposures, causes of infertility, and other potential cancer risk factors. ${ }^{15}$

Lerner-Geva et al. presented a study to evaluate the possible risk for cancer development in a cohort of 2431 women who were treated for infertility with gonadotropins and other fertility drugs in Israel, with over 30 years of follow-up. They calculated the SIR between the observed cancer cases and the expected cancer rates in the general population. The investigators observed 18 cases of ovarian tumors compared to 18.1 expected (SIR 1.0; 95\% CI: 0.59-1.57). Ovarian cancer risk was not found to be elevated and the authors were not able to demonstrate a significant high risk associated with ovulation stimulating treatments. ${ }^{16}$

\section{Conclusion}

The results of this study suggest that fertility drug usage significantly contribute to overall risk of ovarian cancer when adjusting for known confounding factors. In the next years, the incidence of female infertility is expected to increase. A lot of new drugs are under investigation while other recent drugs are already in current use. More study can be done so that the mortality and mobility could be reduced in the society.

\section{References}

1. Tsilidis K, Allen N, Key T, Dossus L, Lukanova A, Bakken K, et al. Oral contraceptive use and reproductive factors and risk of ovarian cancer in the European Prospective Investigation into Cancer and Nutrition. British journal of cancer 2011;105(9):1436-42.

2. Ferraretti AP, Goossens V, Kupka M, Bhattacharya S, de Mouzon J, Castilla JA, et al. Assisted reproductive technology in Europe, 2009: results generated from European registers by ESHRE. Human reproduction 2013;28(9):2318-31.

3. Moorman PG, Palmieri RT, Akushevich L, Berchuck A, Schildkraut JM. Ovarian cancer risk factors in African-American and white women. American journal of epidemiology 2009;170(5):598-606.

4. Tung K-H, Goodman MT, Wu AH, McDuffie K, Wilkens LR, Kolonel LN, et al. Reproductive factors and epithelial ovarian cancer risk by histologic type: a multiethnic case-control study. American Journal of Epidemiology 2003;158(7):629-38.

5. Braem MG, Onland-Moret N, Van Den Brandt P, Goldbohm R, Peeters P, Kruitwagen R, et al. Reproductive and hormonal factors in association with ovarian cancer in the Netherlands cohort study. American journal of epidemiology 2010;172(10):1181-9.

6. Harlow BL, Cramer DW, Bell DA, Welch WR. Perineal exposure to talc and ovarian cancer risk. Obstetrics \& Gynecology 1992;80(1):19-26.

7. Ness RB, Dodge RC, Edwards RP, Baker JA, Moysich KB. Contraception methods, beyond oral contraceptives and tubal ligation, and risk of ovarian cancer. Annals of epidemiology 2011;21(3):188-96.

8. Jordan SJ, Siskind V, Green AC, Whiteman DC, Webb PM. Breastfeeding and risk of epithelial ovarian cancer. Cancer Causes \& Control 2010;21(1):109-16.

9. Tung K-H, Wilkens LR, Wu AH, McDuffie K, Nomura AM, Kolonel LN, et al. Effect of anovulation factors on pre-and postmenopausal ovarian cancer risk: revisiting the incessant ovulation hypothesis. American journal of epidemiology 2005;161(4):321-9.

10. Chandra A, Martinez GM, Mosher WD, Abma JC, Jones J. Fertility, family planning, and reproductive health of US women; data from the 2002 National Survey of Family Growth. 2005.

11. Doyle P, Maconochie N, Beral V, Swerdlow AJ, Tan S. Cancer incidence following treatment for infertility 
at a clinic in the UK. Human reproduction 2002;17(8):2209-13.

12. Rossing MA, Daling JR, Weiss NS, Moore DE, Self SG. Ovarian tumors in a cohort of infertile women. New England Journal of Medicine 1994;331(12):771-6.

13. Schildkraut JM, Schwingl PJ, Bastos E, Evanoff A, Hughes C. Epithelial ovarian cancer risk among women with polycystic ovary syndrome. Obstetrics \& Gynecology 1996;88(4):554-9.

14. Parazzini F, Pelucchi C, Negri E, Franceschi S, Talamini R, Montella M, et al. Use of fertility drugs and risk of ovarian cancer. Human reproduction 2001;16(7):1372-5.

15. Brinton LA, Lamb EJ, Moghissi KS, Scoccia B, Althuis MD, Mabie JE, et al. Ovarian cancer risk associated with varying causes of infertility. Fertility and sterility 2004;82(2):405-14.

16. Liat L-G, Jaron R, Liraz O, Tzvia B, Shlomo M, Bruno L. Are infertility treatments a potential risk factor for cancer development? Perspective of 30 years of follow-up. Gynecological Endocrinology 2012;28(10):80914. 